\title{
Hyperglycaemic memory affects the neurovascular unit of the retina in a diabetic mouse model
}

\author{
Patrick Friedrichs ${ }^{1} \cdot$ Andrea Schlotterer $^{1} \cdot$ Carsten Sticht $^{2} \cdot$ Matthias Kolibabka $^{1}$. \\ Paulus Wohlfart ${ }^{3}$ - Axel Dietrich ${ }^{3}$. Thomas Linn ${ }^{4}$ - Grietje Molema ${ }^{5}$. \\ Hans-Peter Hammes ${ }^{1}$
}

Received: 15 November 2016 / Accepted: 14 February 2017 /Published online: 20 March 2017

(C) Springer-Verlag Berlin Heidelberg 2017

\begin{abstract}
Aims/hypothesis The aim of this study was to evaluate damage to the neurovascular unit in a mouse model of hyperglycaemic memory.

Methods A streptozotocin-induced mouse model of diabetes (C57BL/6J background) received insulin-releasing pellets and pancreatic islet-cell transplantation. Damage to the neurovascular unit was studied by quantitative retinal morphometry for microvascular changes and microarray analysis, with subsequent functional annotation clustering, for changes of the retinal genome. Results Sustained microvascular damage was confirmed by persistent loss of pericytes in the retinal vasculature $\left(\mathrm{PC} / \mathrm{mm}^{2}\right)$ : compared with healthy controls $\left(1981 \pm 404 \mathrm{PC} / \mathrm{mm}^{2}\right)$, the pericyte coverage of the retinal vasculature was significantly reduced in diabetic mice $\left(1571 \pm 383 \mathrm{PC} / \mathrm{mm}^{2}, p<0.001\right)$ and transplanted mice $\left(1606 \pm 268 \mathrm{PC} / \mathrm{mm}^{2}, p<0.001\right)$. Genes meeting the criteria for hyperglycaemic memory were attributed to the cytoskeletal and nuclear cell compartments of the neurovascular unit. The most prominent regulated genes in the
\end{abstract}

Andrea Schlotterer

Andrea.Schlotterer@medma.uni-heidelberg.de

Fifth Medical Department, Medical Faculty Mannheim, University of Heidelberg, Theodor-Kutzer-Ufer 1-3, 68167 Mannheim, Germany

2 Center of Medical Research, Medical Faculty Mannheim, University of Heidelberg, Mannheim, Germany

3 Sanofi-Aventis Deutschland GmbH, Frankfurt am Main, Germany

4 Clinical Research Unit, Centre of Internal Medicine, Justus-Liebig-University Giessen, Giessen, Germany

5 Departmentof Pathology and Medical Biology, Medical Biology section, University Medical Center Groningen, University of Groningen, Groningen, the Netherlands cytoskeletal compartment were Ddx51, Fgd4, Pdlim7, Utp23, Cep57, Csrp3, Eml5, Fhl3, Mapla, Mapklip1, Mnda, Neil2, Parp2, Myl12b, Dynll1, Stag3 and Sntg2, and in the nuclear compartment were Ddx51, Utp23, Mnda, Kmt2e, Nr6a1, Parp2, Cdk8, Srsf1 and Zfp326.

Conclusions/interpretation We demonstrated that changes in gene expression and microvascular damage persist after euglycaemic re-entry, indicating memory.

Data availability The datasets generated during and/or analysed during the current study are available in the GEO repository, GSE87433, www.ncbi.nlm.nih.gov/geo/query/ acc.cgi?token=idmbysgctluxviv\&acc=GSE87433.

Keywords Animal · Microarray · Microvascular disease · Mouse $\cdot$ Retinopathy

$\begin{array}{ll}\text { Abbreviations } \\ 6 \mathrm{~W} / 12 \mathrm{~W} & 6 \text { weeks/12 weeks } \\ \text { DCCT } & \text { Diabetes Control and Complications Trial } \\ \text { DC } & \text { Diabetic control group } \\ \text { NC } & \text { Normal control group } \\ \text { STZ } & \text { Streptozotocin } \\ \text { Tx } & \text { Transplanted }\end{array}$

\section{Introduction}

Hyperglycaemic memory is part of the pathogenesis of diabetic retinopathy $[1,2]$. Perpetuation of oxidative stress, irreversible accumulation of AGEs and hyperglycaemia-induced epigenetic changes are possible underlying mechanisms.

In diabetic dogs, retinopathy develops during euglycaemia after initial hyperglycaemia. In the human retina, the effect of a less-well-controlled period is perpetuated into the period of 
euglycaemic control. In the Diabetes Control and Complications Trial (DCCT), progression to diabetic retinopathy was increased in the conventional treatment group. In the follow-up study, this effect persisted even after equalising $\mathrm{HbA}_{1 \mathrm{c}}$ levels.

Cell culture experiments revealed several important mechanisms of hyperglycaemic memory. The transcription factor $\mathrm{mSin} 3 \mathrm{~A}$ is persistently activated upon short cellular exposure to high glucose, regulating angiopoietin-2, an angiogenic growth factor and regulator of diabetic pericyte dropout [3]. Short exposure to high glucose also induces epigenetic modifications and subsequent downregulation of antioxidant defence proteins (e.g. mediated by the sustained recruitment of SET7 methyltransferase to the NFKB p65 promoter [4]).

Hyperglycaemic memory has mainly been studied in shortterm (cell culture) or long-term animal models, such as the studies by Kowluru [5]. Therefore, the aim of this study was to evaluate a novel model of intermediate-term hyperglycaemic memory using islet transplantation to enable the study of subsequent early structural alterations in the neurovascular unit of the retina in diabetic retinopathy.

\section{Methods}

Animals Male C57BL/6J mice (Charles River, Frankfurt, Germany) were housed under a $12 \mathrm{~h}$ light-dark cycle with free access to food and water. Experiments were conducted in accordance with the Association for Research in Vision and Ophthalmology statement for the Use of Animals in Ophthalmic and Vision Research. The study was approved by the Regional Commission in Karlsruhe, Germany.

Experimental groups Eight-week-old streptozotocin (STZ)induced diabetic mice (diabetic control [DC]), 8-week-old STZ-induced diabetic mice receiving isogenic pancreatic islet transplantation (Tx) after 6 weeks of diabetes (DC+Tx) and age-matched controls (non-diabetic control [NC]) were analysed after 6 weeks $(6 \mathrm{~W})$ and 12 weeks $(12 \mathrm{~W})$. Use of isogenic animals and sterile conditions ensured the absence of immune responses and surgical complications. All mice were euthanised under general anaesthesia.

Diabetes induction Diabetes was induced by intraperitoneal injection of STZ (160 mg/kg body weight; SigmaAldrich, Munich, Germany) in 8-week-old mice. Stable hyperglycaemia was confirmed 7 days after injection by blood glucose $>16.7 \mathrm{mmol} / \mathrm{l}$. Body weight and blood glucose were measured throughout the experiment (BGStar; Sanofi-Aventis, Frankfurt am Main, Germany; limited to $33.3 \mathrm{mmol} / \mathrm{l})$. $\mathrm{HbA}_{1 \mathrm{c}}$ was measured using affinity chromatography (In2it; Biorad, Munich, Germany).
Islet transplantation Transplantation of pancreatic islet cells was performed by the Clinical Research Unit, Giessen (TL). Islets were taken from 8-week-old male C57BL/6J mice (Janvier Labs, Saint-Berthevin, France). Pancreatic islet-cell isolation was performed using pancreatic collagenase digestion and handpicked selection [6]. About 300 islets were injected below the kidney capsule of the recipient mice after 6 weeks of diabetes induction. To support a basal insulin release of the islet graft, insulin-releasing pellets (LinBit; LinShin Canada, Toronto, ON, Canada), each releasing insulin at $0.1 \mathrm{U} / 24 \mathrm{~h}$, were placed subcutaneously below the mid dorsal skin. The number of pellets placed in each recipient mouse, estimated by the targeted reduction of blood glucose levels $(<13.9 \mathrm{mmol} / \mathrm{l})$, was two to four.

Retinal digestion Retinas were isolated after overnight fixation of frozen eyes $\left(-80^{\circ} \mathrm{C}\right)$ in $4 \%$ buffered formalin and were digested using a trypsin-based digestion method. Four to six retinas from each group were analysed morphometrically. In each retina, ten fields were randomly selected using $\times 400$ magnification and CellF analysing software (Olympus, Hamburg, Germany). The cell numbers were normalised to relative capillary density (cell number $/ \mathrm{mm}^{2}$ capillary area).

RNA isolation Frozen eyes were dissected and the retinas were extracted and immediately suspended in Trizol reagent (Invitrogen, Carlsbad, CA, USA). Zirconium oxide beads (1 mm, RNase-free; Next Advance, Averill Park, NY, USA) and a Bullet Blender (Next Advance) were used for retinal homogenisation. RNA was extracted using Trizol. RNA quality was verified using a Bioanalyzer 2100 (Agilent, Santa Clara, CA, USA) and quantity measured by spectroscopy using an Infinite200 PRO NanoQuant System (Tecan, Männedorf, Switzerland).

Gene expression profiling Gene expression profiling $(n=5$ or 6 per group) was performed using Affymetrix GeneChip Mouse Gene 2.0 ST Array. Biotinylated antisense cRNA was then prepared in accordance with a standard labelling protocol. All protocols and equipment were from Affymetrix (High Wycombe, UK).

Bioinformatics A custom CDF Version 19 with Entrez-based gene definitions was used to annotate the arrays. The raw fluorescence intensity values were normalised applying quantile normalisation and robust multi-array average (RMA) background correction. ANOVA was performed to identify differentially expressed genes (SAS JMP10 Genomics v6; SAS Institute, Cary, NC, USA). A false-positive rate of $\mathrm{a}=0.05$ with correction for false discovery rate was taken as the level of significance (GEO: GSE87433). Metabolic memory genes were identified, if they were regulated positively or negatively, in both $\mathrm{NC12W} \rightarrow$ DC12W and NC12W $\rightarrow$ DC+Tx@12W. Analysis of the resulting gene list was performed using DAVID 
bioinformatics database (version 6.7, https://david-d.ncifcrf.gov/) using the categories UP TISSUE and GOTERM CC FAT.

Statistical analysis Metabolic data and pericyte numbers are expressed as mean \pm SEM and were analysed using one-way ANOVA followed by Tukey's multiple comparison post hoc test. Animals were assigned to experimental groups by simple randomisation. Experimenters were blind to group assignment and outcome assessment. No data, samples or animals were excluded from the reported results.

\section{Results}

The average blood glucose level in healthy control mice (NC) was $10.4 \pm 1.9 \mathrm{mmol} / \mathrm{l}$. STZ treatment (DC) resulted in elevation of blood glucose above $33.0 \mathrm{mmol} / \mathrm{l}$. Islet transplantation significantly reduced blood glucose from $31 \pm 3.7 \mathrm{mmol} / 1$ (DC+ Tx@6W) to $8.4 \pm 1.2 \mathrm{mmol} / \mathrm{l}$ (DC+Tx@12W) (Fig. 1a). Changes in $\mathrm{HbA}_{1 \mathrm{c}}$ corresponded to blood glucose levels (data

a

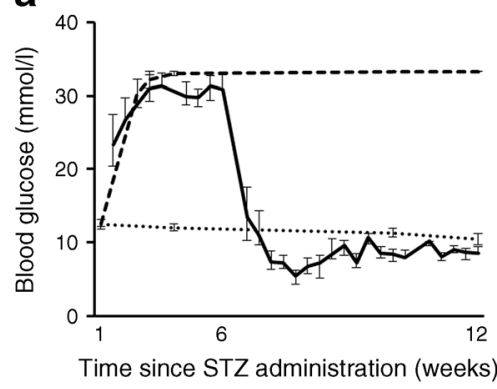

c

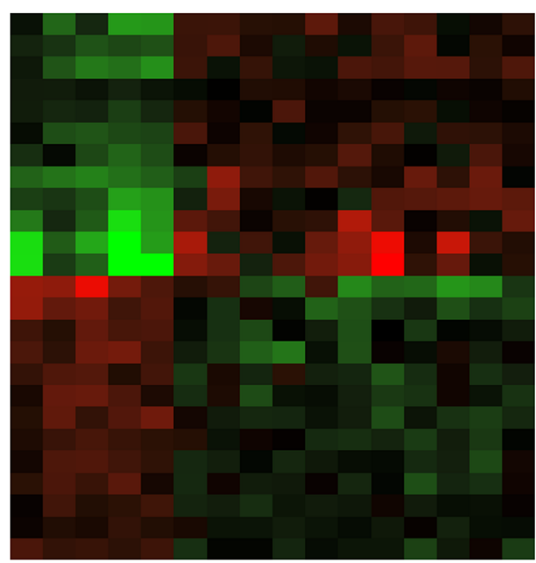

Snord69 Reln Klf6 Tnfrsf17
Mdh1 Sn $\times 3$ Eif3h Tusc5 Clstn2 Snord47 Snord12 Sfxn? Sfxn2 Stag3 Galnt7 Gaint7 Zfp292 Fgd4 Clcn1 KIhI18 KIhl18 Wdr17 Ankhd

Fig. 1 (a) Average blood glucose levels in healthy controls (NC, $n=6$, dotted line), STZ-diabetic mice (DC, $n=6$, dashed line) and STZ-induced diabetic mice receiving islet transplantation ( $\mathrm{DC}+\mathrm{Tx}, n=5$, solid line). Data are expressed as mean \pm SEM. (b) Pericyte (PC) coverage of retinal vasculature in healthy controls $(\mathrm{NC} 12 \mathrm{~W}, n=6)$, STZ-induced diabetic mice (DC12W, $n=6$ ) and STZ-induced diabetic mice receiving islet transplantation (DC+Tx@12W, $n=4)$. Data are expressed as mean \pm SEM. $* * * p<0.001$ vs NC12W. (c) Heatmap of top 25 not shown). Hyperglycaemia was associated with reduced body weight (DC12W 27.7 $\pm 2.3 \mathrm{~g}$ ) whereas the transplanted group recovered normal body weight after receiving islet-cell transplantation (DC+Tx@12W 30.0 \pm 0.8 g; NC12W $32.3 \pm 1.4$ g).

To assess microvascular changes, the number of pericytes per retinal capillary area $\left(\mathrm{PC} / \mathrm{mm}^{2}\right)$ was determined. Compared with healthy controls $\left(1981 \pm 404 \mathrm{PC} / \mathrm{mm}^{2}\right)$, the pericyte coverage of the retinal vasculature was significantly reduced in diabetic mice $\left(1571 \pm 383 \mathrm{PC} / \mathrm{mm}^{2}, p<0.001\right)$ and transplanted mice $\left(1606 \pm 268 \mathrm{PC} / \mathrm{mm}^{2}, p<0.001\right)$ (Fig. 1b). Endothelial cells were not affected by hyperglycaemic conditions or transplantation (data not shown).

To identify genes involved in hyperglycaemic memory, we analysed expression patterns not regulated by euglycaemic reentry, with full genome mRNA arrays. One hundred and twentysix genes were identified as meeting these criteria. The top 25 regulated genes are presented in Fig. 1c; relative expression compared with the $\mathrm{NC12} \mathrm{W}$ group is shown. Clustering analysis upon functional annotation revealed two compartments affected by hyperglycaemic memory: cytoskeleton and nucleus (Fig. 1d,

\section{b}

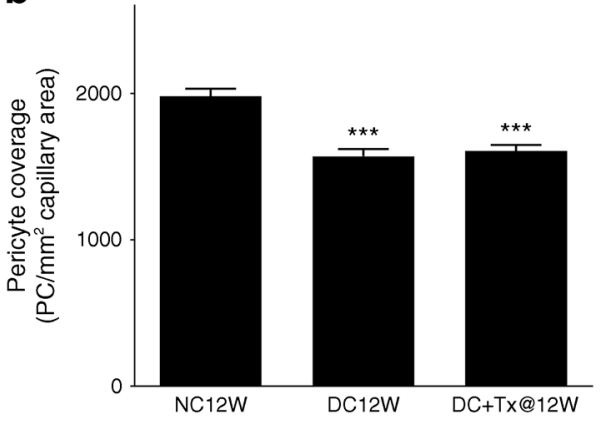

d

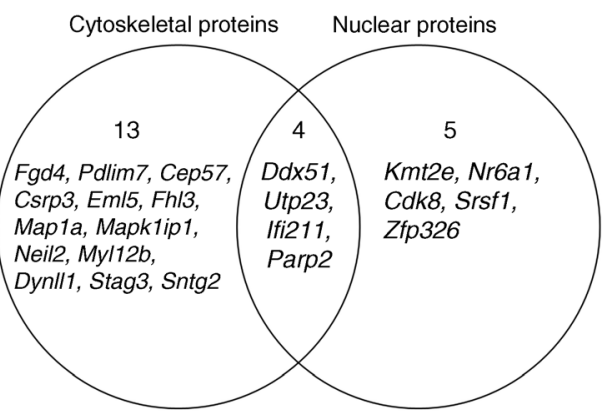

upregulated and downregulated memory genes of healthy controls (NC12W, $n=5$ ), STZ-induced diabetic mice (DC12W, $n=6$ ) and STZ-induced diabetic mice receiving islet transplantation (DC+Tx@12W, $n=5$ ). Colour and brightness indicate relative expression change compared with $\mathrm{NC} 12 \mathrm{~W}$ (green = positive expression change; red $=$ negative expression change). (d) Distribution of regulated memory genes between the cytoskeletal and nuclear compartments 
Table 1). The most prominent regulated genes in the cytoskeletal compartment were Ddx51, Fgd4, Pdlim7, Utp23, Cep57, Csrp3, Eml5, Fhl3, Mapla, Mapk1ip1, Mnda, Neil2, Parp2, Myl12b, Dynll1, Stag3 and Sntg2, and in the nuclear compartment were Ddx51, Utp23, Mnda, Kmt2e, Nr6a1, Parp2, Cdk8, Srsf1 and Zfp326.

\section{Discussion}

In our study, we demonstrated that changes in gene expression and microvascular damage in the neurovascular unit of the retina in a model of diabetes persisted after euglycaemic reentry, indicating hyperglycaemic memory.

Euglycaemic re-entry of islet-transplanted mice was confirmed by blood glucose and $\mathrm{HbA}_{1 \mathrm{c}}$. The reduction in $\mathrm{HbA}_{1 \mathrm{c}}$ levels mimics the effect seen in diabetic patients on intensive insulin treatment and appears to be greater than the reduction following sole insulin treatment in animals, which in rodents bears the disadvantage of glucose variability. However, restoration of euglycaemia did not correct microvascular damage, as reflected by sustained reduction in pericyte coverage of the retinal vascular network. This persistence of microvascular damage is considered to be caused by hyperglycaemic memory. Pericytes are an important component of the neurovascular unit, placed at the intersection of the neuroglial and vascular compartments in the retina and are also known to be the first cell type to be damaged under hyperglycaemic conditions [7].

Genes meeting the criteria for hyperglycaemic memory were attributed to the cytoskeletal and nuclear compartments. The cytoskeleton has been shown to be involved in the pathogenesis of diabetic microvascular complications [8, 9]. More importantly, sustained changes in nuclear factors have been reported to have a large-scale impact on gene expression, resulting in changes in antioxidative defence mechanisms to hyperglycaemic stress [10].

Table 1 Expression changes of cytoskeletal and nuclear proteins

\begin{tabular}{|c|c|c|c|c|}
\hline \multirow[t]{2}{*}{ Gene symbol } & \multirow[t]{2}{*}{ Gene name } & \multirow[t]{2}{*}{ Entrez ID } & \multicolumn{2}{|c|}{ Fold change vs $\mathrm{NC} 12 \mathrm{~W}$} \\
\hline & & & DC12W & DC+Tx@12W \\
\hline \multicolumn{5}{|l|}{ Cytoskeleton } \\
\hline$D d x 51$ & DEAD (Asp-Glu-Ala-Asp) box polypeptide 51 & 69663 & 1.14 & 1.06 \\
\hline $\mathrm{Fgd4}$ & FYVE, RhoGEF and PH domain containing 4 & 224014 & 1.19 & 1.17 \\
\hline Pdlim 7 & PDZ and LIM domain 7 & 67399 & 0.87 & 0.95 \\
\hline Utp23 & UTP23, small subunit (SSU) processome component & 78581 & 0.92 & 0.92 \\
\hline Cep57 & Centrosomal protein 57 & 74360 & 1.10 & 1.11 \\
\hline Csrp3 & Cysteine and glycine-rich protein 3 & 13009 & 0.86 & 0.88 \\
\hline Eml5 & Echinoderm microtubule-associated protein like 5 & 319670 & 1.11 & 1.05 \\
\hline Fhl3 & Four and a half LIM domains 3 & 14201 & 0.84 & 0.92 \\
\hline Mapla & Microtubule-associated protein $1 \mathrm{~A}$ & 17754 & 0.83 & 0.89 \\
\hline Mapklipl & Mitogen-activated protein kinase 1 interacting protein 1 & 69546 & 1.18 & 1.23 \\
\hline Ifi211 & Interferon activated gene 211 & 381308 & 0.87 & 0.95 \\
\hline Neil2 & nei like 2 (E. coli) & 382913 & 1.16 & 1.21 \\
\hline Parp2 & Poly (ADP-ribose) polymerase family, member 2 & 11546 & 1.20 & 1.14 \\
\hline Myl12b & Myosin, light chain $12 \mathrm{~B}$, regulatory & 67938 & 0.89 & 0.90 \\
\hline Dynll1 & Dynein light chain LC8-type 1 & 56455 & 0.85 & 0.89 \\
\hline Stag3 & Stromal antigen 3 & 50878 & 1.22 & 1.14 \\
\hline Sntg2 & Syntrophin, gamma 2 & 268534 & 1.17 & 1.08 \\
\hline \multicolumn{5}{|l|}{ Nucleus } \\
\hline$D d x 51$ & DEAD (Asp-Glu-Ala-Asp) box polypeptide 5 & 69663 & 1.14 & 1.06 \\
\hline Utp23 & UTP23, small subunit (SSU) processome component & 78581 & 0.92 & 0.92 \\
\hline Ifi2 11 & Interferon activated gene 211 & 381308 & 0.87 & 0.95 \\
\hline$K m t 2 e$ & Lysine $(\mathrm{K})$-specific methyltransferase $2 \mathrm{E}$ & 69188 & 1.10 & 1.15 \\
\hline Nr6al & Nuclear receptor subfamily 6 , group A, member 1 & 14536 & 1.13 & 1.06 \\
\hline Parp2 & Poly (ADP-ribose) polymerase family, member 2 & 11546 & 1.20 & 1.14 \\
\hline$C d k 8$ & Cyclin-dependent kinase 8 & 264064 & 1.16 & 1.05 \\
\hline Srsfl & Serine/arginine-rich splicing factor 1 & 110809 & 1.12 & 1.06 \\
\hline Zfp 326 & Zinc finger protein 326 & 54367 & 1.10 & 1.08 \\
\hline
\end{tabular}


Our mouse model of hyperglycaemic memory revealed persistent changes in both gene expression patterns and early structural alterations in the neurovascular unit of the diabetic retina.

Data availability The datasets generated during and/or analysed during the current study are available in the GEO repository, GSE87433, https:/ www.ncbi.nlm.nih.gov/geo/query/acc.cgi?token=idmbysgctluxviv\&acc= GSE87433.

Funding This study was supported by the Deutsche Forschungsgemeinschaft (International Research Training group 1874-1 DIAMICOM and the Deutsche Diabetes Gesellschaft).

Duality of interest The authors declare that there is no duality of interest associated with this manuscript.

Contribution statement PF made substantial contributions to acquisition of data and drafting the article. AS, CS, MK, PW, AD, TL and GM made substantial contributions to analysis and interpretation of data, and revising the article critically for important intellectual content. $\mathrm{H}-\mathrm{PH}$ made substantial contributions to conception and design, and revising the article critically for important intellectual content. All authors gave final approval of the version to be published. H-PH is responsible for the integrity of the work as a whole.

\section{References}

1. The Diabetes Control and Complications Trial (DCCT)/ Epidemiology of Diabetes Interventions and Complications (EDIC) Research Group (2015) Effect of intensive diabetes therapy on the progression of diabetic retinopathy in patients with type 1 diabetes: 18 years of follow-up in the DCCT/EDIC. Diabetes 64: 631-642

2. Engerman RL, Kern TS (1987) Progression of incipient diabetic retinopathy during good glycemic control. Diabetes 36:808-812

3. Yao D, Taguchi T, Matsumura $T$ et al (2007) High glucose increases angiopoietin-2 transcription in microvascular endothelial cells through methylglyoxal modification of mSin3A. J Biol Chem 282:3103831045

4. El-Osta A, Brasacchio D, Yao D et al (2008) Transient high glucose causes persistent epigenetic changes and altered gene expression during subsequent normoglycemia. J Exp Med 205:2409-2417

5. Tewari S, Zhong Q, Santos JM, Kowluru RA (2012) Mitochondria DNA replication and DNA methylation in the metabolic memory associated with continued progression of diabetic retinopathy. Invest Ophthalmol Vis Sci 53:48814888

6. Lai Y, Schneider D, Kidszun A et al (2005) Vascular endothelial growth factor increases functional $\beta$-cell mass by improvement of angiogenesis of isolated human and murine pancreatic islets. Transplantation 79:1530-1536

7. Cogan DG, Toussaint D, Kuwabara T (1961) Retinal vascular patterns. IV. Diabetic retinopathy. Arch Ophthalmol 66: 366-378

8. Chang YC, Chang EY, Chuang LM (2015) Recent progress in the genetics of diabetic microvascular complications. World J Diabetes 6:715-725

9. Anand A, Bammidi S, Bali P (2014) Cytoskeleton dynamics in the retina. Crit Rev Eukaryot Gene Expr 24:255-268

10. Bierhaus A, Schiekofer S, Schwaninger M et al (2001) Diabetesassociated sustained activation of the transcription factor nuclear factor-kappaB. Diabetes 50:2792-2808 